\title{
BOYAS, FAROS Y LA PROVISIÓN VOLUNTARIA DE BIENES PÚBLICOS*
}

\author{
Martín Krause \\ Universidad de Buenos Aires
}

\begin{abstract}
RESUMEN: El debate sobre los faros se ha convertido en un elemento central de la teoría económica, ya que plantea qué arreglos institucionales pueden existir para proveer servicios que tienen limitaciones para excluir a quienes no pagan. En un trabajo fundacional de este debate, Ronald Coase analizó la historia de la provisión de faros en Inglaterra y puso énfasis en el papel de los inversores privados para su construcción y operación. A continuación se originó un debate sobre si podían ser calificados como "privados", siendo que se financiaban con tasas impuestas por el gobierno. Van Zandt entonces clasificó los diferentes arreglos institucionales posibles en cinco categorías, que van desde la total provisión privada hasta la completamente estatal. Él y otros autores afirmaron que no existían casos históricos de bienes públicos con provisión y financiamiento privado con la mera función gubernamental de afirmar derechos de propiedad y el cumplimiento de contratos. Esta investigación muestra que sí existiría un caso de tal naturaleza, hoy en funcionamiento en la costa norte del Río de la Plata, al norte de la ciudad de Buenos Aires. Su
\end{abstract}

Martín Krause. Doctor en administración de la Universidad Católica de La Plata. Profesor titular en la Universidad de Buenos Aires. Miembro de la Mont Pèlererin Society y académico adjunto del Cato Institute. Autor, entre otros, de El foro y el bazar (2014); Economía, instituciones y politicas públicas (2011); y Por el ojo de una aguja: ética, negocios y dinero en el mundo de hoy (2007). Email: mkrause@, derecho.uba.ar.

* El autor quiere agradecer la información recibida de Andrés Galíndez, presidente del CNSI, y Juan Carlos Tollan, presidente de UNEN, así como los comentarios recibidos por los evaluadores anónimos de Estudios Públicos. 
existencia plantea la necesidad de considerar nuevos aspectos de esta discusión fundamental.

PALABRAS ClAVE: bienes públicos, provisión estatal, provisión privada, faros, Coase, Van Zandt.

RECIBIDO: abril 2015; ACEPTADO: agosto 2015.

Clasificación JEL: H11, H41.

\section{BUOYS, BEACONS AND THE VOLUNTARY PROVISION OF PUBLIC GOODS}

ABSTRACT: The debate over lighthouses has become a central feature of economic theory, since it addresses what kind of institutional setting may be able to supply services characterized by limitations to exclude non-payers and the potential of free riding conducts. In a seminal work Ronald Coase reported on the history of lighthouse provision in England and stressed the role of private investors and private funding for their building and operation. A debate ensued on whether this could be qualified as "private" considering they were financed through "light dues" of a coercive nature. Van Zandt afterwards classifies the different institutional settings in five categories ranging from total private provision and financing to complete government supply. He and other authors claimed there are no historical cases of private provision or financing with only government enforcement of property and contract rights. This article shows one such case presently working in the northern coast of the River Plate, in northern Buenos Aires. Its existence raises new issues to be considered.

KEYWORDS: public goods, government supply, private supply, lighthouses, Coase, Van Zandt.

ReCEIVED: April 2015; ACCEPTED: August 2015.

JEL Classification: H11, $H 41$.

os faros han estado iluminando la discusión económica durante al menos los dos últimos siglos de historia. Ronald Coase (1974) analizó las referencias de economistas clásicos como John Stuart Mill, Henry Sidgwick y Alfred Pigou en su contribución a demarcar lo que más adelante se llamarían "bienes públicos", algo que, para términos de este artículo, definiremos como aquellos bienes cuyas características hacen imposible o inconveniente su provisión voluntaria. Estos primeros autores enfatizaron principalmente la característica de la "no exclusión" de los bienes públicos, esto es, la imposibilidad de excluir a 
quienes no quisieran pagar por el servicio, los hoy definidos como free riders. Paul Samuelson (1954) expandió el análisis a otra característica, la "no rivalidad en el consumo", a partir de la cual tampoco sería eficiente excluir, ya que en determinados bienes el consumo que unos realicen no reduce la misma posibilidad por parte de otros. El objetivo de Coase, sin embargo, era presentar una historia que pudiera servir para ampliar nuestra visión de la variedad de arreglos institucionales posibles $(1974,362)$, un objetivo que formaba ya parte de un proyecto de investigación desplegado a partir de numerosos textos (ver, por ejemplo, Coase 1937; 1959; 1960).

El marco institucional para la provisión y financiamiento de estos servicios, sin embargo, parece haber sido diferente para los autores clásicos que para Samuelson, tal vez por las diferencias encontradas en el servicio entre el siglo XIX y el siguiente. Pareciera que Samuelson tuvo en mente tanto la provisión como el financiamiento estatal, puesto que niega la posibilidad de su provisión privada, dado que "un empresario no lo construiría motivado por el lucro" y porque "ésta es, por cierto, el tipo de actividad que los gobiernos normalmente asumirían" (citado por Coase 1974, 358). Mill, al menos, parece haber considerado la financiación a través de tasas y provisión privada. No extraña que lo pensara, ya que ése era entonces el marco institucional para la provisión del servicio de faros en toda Europa, desde que comenzaron a construirse en el siglo XVI (ver Lindberg 2009, para Escandinavia, y Põder 2010, para Estonia).

\section{TRINITY HOUSE, ¿PRIVADA O PÚBLICA?}

El análisis de Coase se basó en la historia del sistema británico entre los siglos XVI y XIX, y el papel que cumpliera el Lord High Admiral, empresarios privados y la Trinity House. Coase describe a esta última como una antigua institución, que supuestamente ha evolucionado a partir de un gremio de marineros y pescadores, lo que coincide con la descripción que aún hoy esta organización presenta de sí misma:

Se afirma a menudo que los orígenes de Trinity House se remontan a un gremio benéfico de marineros samaritanos establecido por el arzobispo Stephen Langton en el siglo XII. 
El primer registro oficial es el otorgamiento de una carta real por Henry VIII el 20 de mayo de 1514 a una fraternidad de marineros llamada Gremio de la Santa Trinidad "para que puedan regular el pilotaje de barcos en los arroyos del rey". En momentos de su creación, este gremio caritativo poseía un importante salón y asilos, cerca del muelle naval en Deptford, sobre el río Támesis. El otorgamiento de esta carta fue resultado de una petición que se le presentara un año antes del reglamento "Maestros, capitanes y marineros de la Armada Real en el Támesis y otros lugares", de fecha 13 de marzo de 1513. Aunque no han quedado registros oficiales escritos para corroborar la evidencia, dos lápidas en la iglesia Leigh en Essex indican la existencia de una organización relacionada con el bienestar de los marinos en las costas de Inglaterra en el siglo XV. Cada una de las lápidas muestra el nombre de un "Hermano de la Trinidad yace debajo". (Trinity House)

Entre las funciones actuales de la Trinity House está la de ser la Autoridad General de Faros (GLA, por su sigla en inglés) para Inglaterra, Gales y las islas del Canal de la Mancha, proveyendo ayudas para la navegación, desde faros y boyas a tecnología satelital para la navegación. También inspecciona las ayudas locales provistas por las autoridades portuarias. Sus gastos los cubre el Fondo General de Faros, formado con ingresos provenientes de las tasas que paga la navegación comercial. Las tasas son fijadas por el Departamento de Transporte y se pagan por tonelada neta solamente por los buques comerciales y en las primeras seis visitas a puertos británicos por año. La Trinity House todavía mantiene su actividad caritativa original, aunque separada de sus actividades como autoridad de faros.

Coase describe, y Trinity House confirma, que muchos faros fueron construidos por privados, operados y mantenidos a través de licencias obtenidas desde la Trinity House o la misma corona. "Los peajes eran cobrados en los puertos por agentes (que podían actuar para varios faros), quienes podían ser individuos privados pero eran generalmente funcionarios de aduanas" (Coase 1974, 364). Todos estos faros privados fueron adquiridos compulsivamente luego de que se aprobara una ley en 1836, y se colocaron bajo la administración de Trinity House. El argumento entonces - $y$ hasta hoy sostenido por Trinity House - fue que la confiabilidad de muchos de estos faros privados dejaba mucho 
que desear, aunque Coase arguye que el motivo real era una "búsqueda de rentas", la expectativa de los usuarios de que, al alejar a los privados, bajarían las tasas, un argumento recientemente enfatizado por Carnis (2013).

El principal objetivo del artículo de Coase (1974) fue cuestionar la idea, generalmente aceptada, de que la provisión y financiamiento de bienes públicos sólo puede resolverse por vía estatal, para lo cual se valió del ejemplo de los faros. Así, no descartó otras posibilidades de provisión privada de bienes públicos, afirmando que el relato sobre el sistema británico de faros así lo revela (Coase 1974, 375).

Es de esta manera como describe al sistema como "privado", aunque claramente se refiere a la provisión, no al financiamiento:

La temprana historia muestra que, contra la creencia de muchos economistas, el servicio de faros puede ser provisto por la empresa privada. En esos días, los dueños de buques y armadores podían solicitar a la Corona que permitiera a un individuo particular construir un faro y aplicar una tasa (específica) a los barcos que se beneficiaran con él. Los faros eran construidos, operados, financiados y propiedad de individuos particulares, quienes podían venderlos o legarlos. El papel del gobierno estaba limitado al establecimiento y aplicación de derechos de propiedad sobre el faro. Los cargos eran recolectados en los puertos por agentes de los faros. El problema de la aplicación no era distinto para ellos que para otros proveedores de bienes y servicios a los dueños de barcos. Los derechos de propiedad eran inusuales solamente en que estipulaban el precio que podía cobrarse. (Coase 1974, 375)

La mención a que los faros eran "financiados" por individuos privados se refiere al capital original invertido para construirlos, no a su operación y mantenimiento, que sabemos provenía de las tasas. No obstante, esta mención desató un intenso debate si se podía llamar "privado" a dicho marco institucional.

El primero en arrojar una piedra fue Van Zandt, al argüir que el concepto de "empresa privada [...] difícilmente captura la realidad de la provisión de servicios de faros", ya que "el gobierno jugó un papel substancialmente más importante en la provisión de servicios de faros de lo que el término 'privado' de Coase sugiere" (Van Zandt 1993, 48). Más adelante, tanto Bertrand (2006) como Barnett y Block (2007) 
plantearon esa misma preocupación, aunque desde dos perspectivas diferentes: Bertrand muestra que fueron organizaciones caritativas las que proveían y financiaban los faros, pero no las llama "empresas privadas" porque no estaban motivadas por el lucro; mientras que Barnett y Block sostienen que el sector caritativo de la economía es privado, ya que es el resultado de acciones voluntarias, como aquellas de empresas que maximizan ganancias.

Block y Barnett $(2009,2)$, en base a su definición, definen el caso británico tal como lo presenta Coase como una oferta "gubernamental, no basada en el mercado". Lo mismo hacen Lindberg $(2009,23)$ y Block (2011, 51).

Todos ellos, a su manera, consideran cómo podría funcionar un sistema "privado voluntario". Van Zandt (1993), sin embargo, desarrolla una clasificación menos drástica siguiendo las ideas de un "continuo" de arreglos institucionales entre uno puro privado voluntario y otro puro gubernamental compulsivo, donde existe una gama de distintas alternativas. Así las define Van Zandt (1993, 56):

1. Provisión privada sin protección gubernamental de derechos de propiedad y contratos;

2. Provisión privada con protección gubernamental de sólo derechos de propiedad y contratos;

3. Provisión privada con un gobierno que establece tasas, otorga monopolios y asegura la recaudación de tasas específicas para usuarios;

4. Provisión gubernamental financiada con el cobro de tasas específicas, y

5. Provisión gubernamental financiada con recursos fiscales generales.

Samuelson parece presentar el ejemplo de los faros como uno de bienes públicos en las categorías 4 o 5 ; Coase describe al sistema británico como la opción 3. La mayoría de los países tienen sistemas que se ubican en las categorías 3 o 4, y tales son los casos, por ejemplo, de la United States Coast Guard (USCG), Canadian Coast Guard o Australian Maritime Safety Authority. En los Estados Unidos, la USCG se ha concentrado en balizas automáticas y el mantenimiento de ayudas a la navegación, como boyas de estructuras de acero por sobre las linternas usuales de los faros tradicionales. Estos fueron descuidados y vandalizados hasta que se entregaron en concesión a sociedades históricas lo- 
cales y otras organizaciones o fueron vendidos como propiedad superflua. Chile es un ejemplo de la categoría 5: los faros y todas las ayudas a la navegación son financiados por el tesoro nacional y administrados por el Servicio de Señalización Marítima, dependiente de la Armada.

En definitiva, nos encontramos con arreglos en las categorías 3 a 5, y algunos autores imaginan cómo sería posible alguna de las otras opciones. Este artículo presenta una de ellas, un caso dentro de la categoría 2.

\title{
UN SISTEMA PRIVADO VOLUNTARIO
}

La posibilidad de un sistema privado voluntario es explorada por Van Zandt (1993) y Block y Barnett (2009). Van Zandt (1993, 56) dice:

\begin{abstract}
No hay, sin embargo, ejemplos de faros operando en un mundo privado puro (categoría 2), en el cual el gobierno solamente protegiera los derechos de propiedad y acuerdos consensuados. En casi todos los casos, el gobierno ha hecho mucho más. Ha otorgado al propietario del faro un monopolio sobre la provisión de servicios de faro en una ubicación específica; ha establecido y aplicado un esquema fijo de "tasas"; y ha asistido al operador de faros en el cobro de esas tasas a capitanes de barcos que no hayan consentido. Las únicas excepciones a esta generalización han sido los faros provistos por entidades religiosas o personas; en esos casos, si bien el gobierno no siempre estaba involucrado, es probable que se utilizaran mecanismos socialmente coercitivos para superar los particulares problemas del faro.
\end{abstract}

Ahora bien, la coerción social es diferente de la coerción gubernamental. Esta última incluye la fuerza o la amenaza del uso de la fuerza en un cierto territorio, una definición que está implícita en el argumento de Block y Barnett. Por ende, ya sea que esas entidades religiosas o personas particulares desafien tal monopolio (como lo hace un criminal, una mafia, una insurrección o un poder extranjero) o no, en último caso las contribuciones que reciben son voluntarias, ya que estas entidades no pueden enviar a la cárcel a quienes no pagan, embargar sus cuentas bancarias ni requerir poderes al gobierno para hacerlo.

Van Zandt considera cómo la tecnología podría convertir una situación de "no exclusión" en una donde la exclusión es posible y, por lo tanto, también la provisión privada. Si los emprendedores no pueden 
excluir a los free riders, la provisión de un cierto bien o servicio no sería posible en base al lucro o podría llevar a un consumo excesivo del recurso. El típico ejemplo es el del ganado vacuno, amenazado con extinguirse hasta que en un determinado momento se introdujeron los derechos de propiedad y los alambrados.

Van Zandt reconoce que podría haber formas de cobrar tasas sobre una base contractual, aunque no se desarrollaron durante la historia de los faros. Especula que con la existencia de la radio, los faros podrían encenderse a pedido; esto es, el capital del barco llamaría al propietario del faro para que lo encendiera a cambio de un determinado pago. Es menos probable, considerando la tecnología moderna, su otra alternativa: colocar empleados en pequeños botes a lo largo del límite de alcance de la luz del faro para que, cuando un barco se aproxime, llegar a un acuerdo y así solicitar al operador del faro que lo encienda. Los ejemplos pueden parecer extraños o inocentes luego de tantos años de innovación tecnológica, pero Van Zandt está en lo correcto al señalar que el problema de exclusión es contingente. Concluye, correctamente, que los costos dependen de las tecnologías existentes y que cuando una tecnología se vuelve rentable los emprendedores encontrarán valioso proveer el bien. Por lo tanto, los bienes públicos lo son solamente dentro de un cierto contexto legal y tecnológico.

Block y Barnett mencionan otras posibilidades. Una de ellas son los seguros. Como una navegación más segura daría como resultado tasas de seguro más bajas, los dueños de los barcos estarían interesados en conseguir los servicios de faros y otras ayudas a la navegación. "Nadie puede estar seguro de los detalles, pero parece muy razonable pensar que los dueños de barcos, los pilotos o los aseguradores, o una combinación de todos ellos, podría formar una organización para proveer dichos servicios" (Block y Barnett 2009, 4).

Otra alternativa es la "presión moral". Los dueños de los faros podrían dar una publicidad negativa de aquellos que son free riders de sus servicios o podrían establecer una asociación voluntaria. Sostienen que "sí, por supuesto, así como los carteles tienden a derrumbarse debido a desafíos tanto internos como externos, esos arreglos podrían no ser definitivos. Pero pueden funcionar en algunos casos donde los costos de transacción sean bajos y una cierta ruta comercial sea utilizada por unos pocos" (Block y Barnett 2009, 3). El siguiente es un caso de este preciso arreglo institucional. 


\section{BOYAS PRIVADAS EN LA COSTA NORTE DEL RÍO DE LA PLATA}

El río Paraná fluye por unos 2.500 kilómetros desde Brasil hasta la cuenca del Río de la Plata, donde se encuentra con el río Uruguay y se divide en miles de ríos, riachos e islas que forman el delta. La ciudad de Buenos Aires se estableció en la costa occidental del Río de la Plata y creció hacia el norte, hacia la región del delta, con suburbios llenos de actividad náutica, tanto comercial como de placer y deportiva.

El barroso río Paraná que crea y extiende el delta tiende también a reducir la profundidad de las vías navegables del Río de la Plata, haciendo dificultosa la navegación y posibilitando el varamiento de barcos. Así, las ayudas a la navegación son esenciales en estas aguas poco profundas. Estos apoyos son responsabilidad formal de una agencia gubernamental dentro del Ministerio de Defensa, llamada Servicio de Hidrografía Naval (SHN), que es financiada con recursos del presupuesto general, por lo que podría caer en la categoría 5 de Van Zandt.

Sin embargo, el estudio y la evaluación de marcos institucionales es una instancia del análisis comparativo entre alternativas del mundo real, no de un mundo ideal. De otra forma caemos en la llamada "falacia de Nirvana" (Demsetz 1969, 1). ${ }^{1}$ Tanto Coase $(1974,362)$ como Van Zandt $(1993,57)$ lo enfatizan. Y el "mundo real" de la provisión en la costa norte del Río de la Plata mostró que el financiamiento estatal solamente se ocupaba de las ayudas a la navegación y la profundidad en las vías utilizadas por grandes barcos comerciales, y terminó por desatender las vías costeras, principalmente utilizadas por barcas para el transporte de arena o madera, veleros y barcos a motor deportivos y recreativos.

En 1989, sin servicios de GPS disponibles aún y en un contexto de creciente navegación recreativa, la costa norte del Río de la Plata no contaba prácticamente con ninguna boya o baliza. Sucesivos gobiernos nacionales con jurisdicción sobre el Río de la Plata habían prestado atención al denominado canal Mitre, algo más alejado de la costa que el canal Costanero al que haremos referencia especial aquí. No es de extrañar esta atención al canal Mitre, ya que éste vincula al Río de la Plata con el río Paraná, tiene mucha actividad comercial y permite la

${ }^{1}$ Merece transcribirse la definición de Demsetz: "Esta visión que ahora prevalece implícitamente en muchas políticas públicas presenta a las alternativas relevantes como una elección entre un arreglo ideal y otro existente e 'imperfecto'. Este enfoque de Nirvana difiere mucho de uno basado en el análisis institucional comparativo, en el cual la elección relevante es entre arreglos institucionales reales". 
salida de buena parte de las exportaciones agrícolas argentinas desde los puertos privados de la zona norte de la Provincia de Buenos Aires, así como de ciudades importantes como Rosario o Santa Fe. Esta "atención" al canal Mitre, junto con la necesidad de su dragado para permitir la navegación de buques de gran calado, derivó en un problema para los usuarios del canal Costanero. En éste último la navegación es principalmente deportiva o de placer. Su mantenimiento — dragado y señalización - no solamente fue olvidado por las autoridades respectivas, sino que además el desecho dragado del Mitre generó un peligro adicional en los fondos, debido a la acumulación de tierra. Agréguese a este problema, la suma de cascos a pique sin balizar y una total desactualización de los datos de batimetría (profundidad del agua) y balizamiento en las cartas náuticas. La escasa actividad comercial en este canal - buques areneros, en lo esencial- modificó su recorrido para preferir el canal Mitre, pero las embarcaciones deportivas o de placer no podían evitar el canal Costanero debido a que sus muelles se localizan en sus orillas o en vinculación directa a éste.

Ese "fracaso" del gobierno desató la iniciativa emprendedora. En 1989, los presidentes de dos clubes náuticos —el Yacht Club Argentino y el Club Náutico San Isidro- acordaron establecer una nueva organización, sin fines de lucro, que incluyera a todos los clubes náuticos y marinas privadas del área. El primer paso fue cerrar un acuerdo con el Servicio de Hidrografía Naval para llevar a cabo un informe batimétrico realizado por personal de la agencia gubernamental, y cuyos costos extraordinarios fueron financiados por estos dos clubes privados, promotores de la nueva asociación. El resultado fue una carta náutica - llamada UNEN 1-, luego ampliamente utilizada y fusionada con otras cartas gubernamentales que fueron actualizadas. Es decir, hubo un primer paso efectivo, con el que las dos instituciones emprendedoras "señalizaron" hacia los demás clubes y marinas su compromiso así como el potencial beneficio de las propuestas actividades conjuntas.

Creada la organización sin fines de lucro, llamada Unión de Entidades Náuticas (UNEN), ${ }^{2}$ se comenzó a fondear las boyas UNEN 1, UNEN 2 y UNEN 3; también se repararon las balizas 7 a 10 y otras ayudas costeras que ahora permitían a los barcos cruzar el canal Costero con mayor seguridad. Más tarde se localizaron distintos restos de

\footnotetext{
${ }^{2}$ Ver http://www.boyadounen.com.ar/.
} 
naufragios. El Club Náutico San Isidro (CNSI) donó una embarcación para realizar estas operaciones. La primera boya fue llamada "Pumper Nic", financiada por una cadena de comida rápida que entonces usaba ese nombre. Luego se renombró UNEN 1 y la organización no ha vuelto a usar publicidad en estos instrumentos, porque las autoridades han requerido que las boyas autorizadas para su colocación tengan las mismas características que tienen las boyas gubernamentales.

En 1994 se construyó una nueva embarcación para realizar estas operaciones en el astillero del CNSI, costeada por esa institución. En ese momento se introdujo un nuevo sistema de financiamiento, con una tasa de "un peso por barco" para cada uno de los clubes miembros. Se posicionaron nuevas boyas en 1996, y la UNEN obtuvo ese año su estatus de asociación civil, en tanto que la embarcación y una lancha adicional se convirtieron en su propiedad.

Hasta el momento de redactar este artículo, a mediados de 2015, la UNEN ha continuado estableciendo ayudas y manteniendo y mejorando las existentes. El sistema funciona de la siguiente manera: la propiedad de las vías navegables es gubernamental, la UNEN solicita permiso para el posicionamiento de una nueva boya al SHN, brindando los detalles precisos de la ayuda y su ubicación; luego la coloca e informa al SHN, que la agrega a la carta náutica. Por lo tanto, tanto el financiamiento como la provisión, excepto la posición en el agua, son privados. ¿Calificaría esto dentro de la categoría 2 de Van Zandt?

Parece ser un caso, uno que su estudio no pudo encontrar: "La única estructura conspicuamente ausente es la provisión privada con una participación estatal mínima (categoría 2)" (Van Zandt 1993, 58). ${ }^{3}$ La

${ }^{3}$ Podría discutirse si el ejemplo de balizas y boyas aquí planteado resulta distinto del caso de los faros analizado por los autores clásicos, de los que Van Zandt no encontrara una solución de tipo 2. Sugiero que no son diferentes en los términos clásicos de la definición de bienes públicos, es decir, de exclusión y no rivalidad en el consumo, que se aplican a ambos. Existen diferencias en cuanto al número de usuarios, sus características y la intensidad de uso, pero en este caso uno parece compensar a otro. En el caso de los faros analizado por Coase son pocos usuarios, pero con supuesta alta valoración de los servicios de los faros, dada su actividad comercial, aunque baja intensidad de uso; en el de las balizas y boyas de la costa norte del Río de la Plata la valoración puede que sea menor, ya que no son barcos de uso comercial, con intensidad de uso algo mayor. La menor valoración parece compensarse con el uso más intenso y su agrupación en clubes reduce el número de negociadores a uno, lo que tal vez es similar al de armadores de buques que Coase estudió en el origen del sistema de señalización de la corona británica. 
UNEN también ubica balizas en tierras privadas, tanto de los mismos clubes miembros como de otros propietarios.

Los clubes miembros de la UNEN son 32, más 4 socios adherentes. Entre ellos tienen unos seis mil barcos-usuarios. El free riding de los mismos clubes es fácilmente controlado: Google Earth les permite controlar el número de barcos en cada uno de ellos. Los clubes cobran a cada uno de los propietarios de barcos por el servicio de amarra, lo que puede incluir la tasa de la UNEN en forma explícita o no. No obstante, el cargo es un porcentaje mínimo de la tasa de amarra. ¿Hay free riders? Los directivos de la UNEN estiman que hay unos doce mil barcos en la región, casi todos ellos en marinas y guarderías privadas, aunque algunas de éstas son socias. Esas empresas privadas han mostrado una típica conducta free rider: aceptan los beneficios de los servicios pero han pospuesto o rechazado el pago con diferentes pretextos. Es posible que esa conducta prevalezca en las marinas porque en ellas se guardan principalmente lanchas a motor, de muy poco calado y, por lo tanto, menos expuestas (aunque no exentas) a los riesgos que dieron origen a estas acciones; mientras que en los clubes náuticos predominan los veleros, con un calado mayor y, por lo tanto, usuarios más interesados en los servicios de señalamiento. Ambos usuarios, de clubes náuticos y de marinas, usan las mismas rutas y, si bien no existen datos al respecto, puede suponerse que en la misma intensidad.

¿Acaso la existencia de free riders condena a las soluciones de categoría 2 como ineficientes? Ése es el argumento de Bertrand (2006), aunque, como es habitual en la economía neoclásica, el ideal óptimo está sujeto a la falacia de Nirvana. En el análisis institucional comparativo las figuras ideales sirven para comprender cómo la realidad no es, una vez que se remueven algunos de sus supuestos y se centra la atención en los arreglos existentes.

Block y Barnett consideran este punto, y el caso de la costa norte del Río de la Plata está más cerca de lo que ellos plantean cuando tratan los mecanismos que podrían utilizar los aseguradores para superar este problema $(2009,4)$ :

¿Necesita alguna de estas amenazas, o todas ellas, eliminar a todos los free riders posibles? No. Hay muchas empresas que continúan operando sin poder excluir a todos los receptores de 
cualquier beneficio externo concebible. Por ejemplo, mucho antes de que el gobierno comenzara a subsidiar a la educación superior en los Estados Unidos había miles de estas instituciones enviando ciudadanos educados a la sociedad, beneficiando no sólo a las instituciones y a los estudiantes, sino también a la sociedad en general. De forma similar, los propietarios de faros bien podrían continuar en el negocio sin recibir pagos de todas y cada una de las personas que se benefician de sus actividades comerciales.

Los clubes en sí mismos son un mecanismo institucional que reduce el problema de free riding, ya que es más factible obtener el acuerdo de 36 clubes de lo que sería obtenerlo de los seis mil propietarios de embarcaciones si no formaran parte de ellos. Ese acuerdo entre clubes se facilita, además, por la continua relación que ellos mantienen entre sí, tanto para considerar problemas que tengan en común, como también de la simple interacción que producen las actividades deportivas (regatas). Esta interacción va generando vínculos que reducen los costos de transacción.

Nuevamente, los resultados de la UNEN deberían compararse con la situación existente antes de 1989, bajo la estricta responsabilidad gubernamental. ¿Sería una solución de la categoría 3 de Van Zandt — provisión privada con un gobierno que establece tasas, otorga monopolios y asegura la recaudación - más eficiente? Podría argumentarse que si se aplicara una tasa obligatoria a esos 12 mil free riders, algo que el gobierno podría hacer con su poder de coerción, se generarían más recursos y se abriría la puerta a más servicios. Pero, por supuesto, ésta es una alternativa hipotética. Y bien puede suceder que el lobby y el favoritismo político terminen financiando otro tipo de gastos no relacionados. La UNEN ha resistido esta tentación hasta el momento y no ha requerido la intervención gubernamental.

Algo de esta naturaleza ocurrió con otra obra en la región: el dragado del canal Costanero. El gobierno local, la Municipalidad de San Isidro, implementó un canon que pagan los clubes en proporción a la cantidad de barcos en cada uno de ellos. Sin embargo, hoy son los mismos clubes los que tienen que ocuparse de esta tarea y, luego de hacerla, reciben una devolución pequeña de los costos involucrados, aunque 
la obra es luego presentada como un ejemplo de colaboración "públicoprivada". 4

Otra solución hipotética al problema de los free riders sería algún desarrollo tecnológico-empresarial que permita su exclusión. Algunos han mencionado la posibilidad de que las boyas y balizas se enciendan cuando son activadas por medio de un código, a través de teléfonos celulares o radio. Soluciones como éstas tienen una larga historia, y van desde el alambrado para determinar la propiedad de la tierra y el ganado, hasta la acuicultura (donde se delimitan porciones del océano, lagos o ríos) o el encriptado de señales satelitales que deben ser decodificadas por el usuario que paga.

Foldvary considera una innovación "institucional", a través de la asignación de derechos de propiedad en las vías de agua: "Si el área acuática cercana al faro fuera un espacio de propiedad privada, o si la agencia gobernante asignara tales derechos, entonces todo el que ingresara sería un invasor a menos que fuera explícitamente bienvenido. Las señales de radar, sonar y electrónicas pueden detectar la presencia de invasores, y alertarles por radio que están ingresando en una zona privada, que requiere un pago. Si el bote o barco persiste en su ingreso, entonces se señaliza y el propietario privado podría multar al invasor más allá de los cargos habituales" (2003, 3). En este caso, el cobro de la tasa por servicios podría ser realizada por la UNEN, si tuviera el derecho de excluir a quienes no paguen. Existe, de hecho, una solución de este tipo en las cercanías: el canal comercial del río Paraná ha sido concesionado a una empresa privada que cobra peaje a los usuarios.

Otra posibilidad, totalmente diferente, es que tanto las boyas como las balizas se vuelvan obsoletas a través del uso generalizado del GPS y el piloto automático.

${ }^{4}$ Por ejemplo: http://www.periodicoparatodos.com.ar/actualidad/nota_286. htm; http://msiprensa.blogspot.com.ar/2010_04_18_archive.html; http://sanisidro. gob.ar/media/2012/10/BOLETIN-OFICIAL-N\%C2\%B0-779-_2da.-de-ENERO_. pdf (Decreto 110); http://elnavegantedeltatour.blogspot.com.ar/2013/04/tasa-porfondeaderos-y-mantenim-iento.html; y http://sanisidro.gob.ar/media/2013/01/ ORDENANZA-FISCAL.pdf (pág. 163 en adelante). 


\section{OTROS EJEMPLOS EN LA ZONA}

La costa norte del Río de la Plata muestra también otros ejemplos de provisión y financiamiento privado de bienes considerados como "públicos".

El Puerto de San Isidro es propiedad del gobierno de la Provincia de Buenos Aires y, como muchos otros, se encuentra desatendido y abandonado. El canal que permite ingresar en él, sin embargo, es utilizado por distintos clubes náuticos y por la guardia costera nacional, llamada Prefectura Naval (ver foto 1). Pese a esto, su dragado y mantenimiento son realizados por el Club Náutico San Isidro (CNSI), ubicado en el margen izquierdo de la boca del canal hacia el Río de la Plata, con su propio presupuesto y alguna ayuda eventual de la Prefectura. Este dragado comenzó en 1922 y continuó en los años siguientes gracias a la donación de un bote por el gobierno local y a la donación de bombas por una compañía importadora. Muchos años después, la UNEN construyó una baliza en la desembocadura del canal.

Este dragado es de fundamental importancia no solamente para los barcos de los socios del CNSI, sino también para los del Club de Veleros de San Isidro — que se encuentra en la zona—, muchos otros propietarios de barcos amarrados en el puerto con permiso municipal y la misma Prefectura Naval, cuyos propios barcos no podrían salir del puerto si no se realizara esta tarea de dragado. Incluso, en más de una ocasión las autoridades de esta agencia gubernamental han solicitado al CNSI el dragado del canal, ya que sus embarcaciones se encontraban con problemas para salir hacia el Río de la Plata. Es decir, tenemos aquí una organización privada realizando el mantenimiento de una propiedad pública, para su propio uso, por cierto, pero también para el de otros y de la misma agencia gubernamental de control de las costas y ríos.

El espigón rompeolas del canal tiene también una larga historia de provisión privada. Fue construido por el CNSI en 1915. El Informe de la Comisión Directiva del Club en aquel año dice:

... se decidió la construcción de un espigón rompeolas. La Comisión intentó por todos los medios conseguir el apoyo de los gobiernos nacional y provincial, proveyendo las rocas necesarias para este trabajo, pero fue imposible, y considerando el reducido presupuesto disponible solicitó el transporte a distintas empresas, 
aunque fue difícil pagar por el material. Finalmente, gracias a la gratitud del socio Arturo O'Connor, quien vendió las rocas a su costo, pagando él mismo los impuestos y la mano de obra, conseguimos 435 toneladas para realizar el trabajo. (CNSI 2011)

La construcción del espigón por parte del CNSI se explica porque su existencia deriva en un beneficio directo para este club, ya que éste, en el margen occidental del canal que conecta el Puerto de San Isidro con el Río de la Plata, protege su propia costa en la orilla opuesta. No obstante, esa construcción genera también beneficios a los demás clubes con sede dentro de la zona del puerto e, incluso más, es aprovechado por la población como lugar de pesca recreativa. De esta forma, el gobierno municipal puede ofrecer un lugar de acceso gratuito a la orilla del río, para la pesca o la recreación, y la Prefectura Naval se favorece con una barrera que protege al canal de salida del puerto por el que inevitablemente tienen que navegar sus propios barcos. Nuevamente, hay free riders sobre los beneficios que genera el espigón, que incluyen al mismo gobierno y su Prefectura, pero en este caso el beneficio directo al CNSI ha sido suficiente como para generar su construcción.

Foto 1: CANAL DE PUERTO SAN ISIDRO

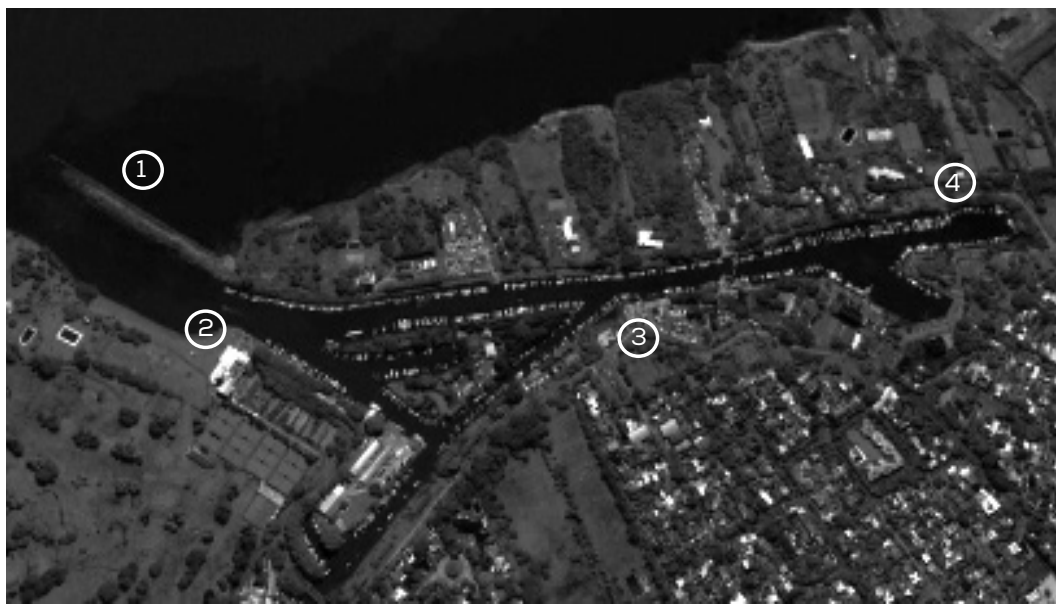

1: Espigón rompeolas

2: Club Náutico San Isidro

3: Prefectura Naval

4: Puerto San Isidro. 
Las diferentes soluciones desarrolladas para el boyado UNEN y para el canal de acceso al puerto podrían explicarse por el grado de concentración de los beneficios, ya que en el primer caso los beneficiados directos eran muchos (los clubes miembros de UNEN) y, en el segundo, el beneficio principal recaía en uno solo (el CNSI).

\section{ALGUNAS CONCLUSIONES}

El debate sobre los faros se ha convertido en un elemento central de la teoría económica, ya que refiere a las características de distintas asignaciones de derechos de propiedad y qué tipo de marco institucional puede permitir la provisión de servicios caracterizados por limitaciones para excluir a quienes no paguen (lo que genera potenciales conductas de free riders).

Dichos marcos deben tomar en cuenta cuestiones relacionadas con el financiamiento y la provisión, y usualmente se ha considerado que las opciones posibles para este tipo de servicios se encontraban dentro de las categorías 3 a 5 en la clasificación de Van Zandt, es decir, donde el financiamiento es compulsivo y la provisión, privada hasta donde el financiamiento y la provisión son gubernamentales. Aunque algunos autores han considerado soluciones hipotéticas que pudieran ser clasificadas en la categoría 2, e incluso en la 1, no se pensaba que existieran casos de ese tipo y tan sólo se mencionaban algunos en la antigüedad, para los cuales no existía demasiada evidencia histórica.

El caso de la UNEN en la costa norte del Río de la Plata presenta un ejemplo vivo, cuyo funcionamiento puede ser evaluado en el presente y podrá serlo en el futuro. Llena el vacío de casos para la categoría 2, la que, pese a ser descartada por muchos autores, muestra su factibilidad. No es una situación perfecta, por supuesto, pero es superior a la previamente existente.

Este caso deja pendientes algunas preguntas de investigación para el futuro: ¿Será necesario resolver finalmente el problema de los free riders o continuará la solución voluntaria, como en el presente? ¿Surgirá un nuevo arreglo institucional que reemplace al actual para reducir a los free riders? ¿O lo hará el avance tecnológico? ¿Evolucionará el presente arreglo hacia otro con mayor definición de derechos de propiedad 
privada? Finalmente, ¿cuánto de esta solución de categoría 2 debe su existencia al fracaso de la categoría 5 ?

El financiamiento y la provisión de ayudas a la navegación por parte de la UNEN en la costa norte del Río de la Plata nos presenta un laboratorio donde se podrá evaluar la evolución de un determinado marco institucional.

\section{REFERENCIAS CITADAS}

Barnett, William \& Walter Block. 2007. "Coase and Van Zandt on Lighthouses". Public Finance Review 55 (6): 710-33.

Bertrand, Elodie. 2006. "The Coasean Analysis of Lighthouse Financing: Myths and Realities". Cambridge Journal of Economics 30: 389-402.

Block, Walter. 2011. "Rejoinder to Bertrand on Lighthouses". Romanian Economic Business Review 6 (3): 49-67.

Block, Walter \& William Barnett. 2009. "Coase and Bertrand on Lighthouses". Public Choice 140 (1-2): 1-13.

Carnis, Laurent. 2013. "The Provision of Lighthouses Services: A Political Economy Perspective”. Public Choice 157 (1-2): 51-6.

Club Náutico San Isidro, CNSI. 2011. "Rasgos de nuestra costa". Náutico 277 (agosto).

Coase, Ronald H. 1937. "The Nature of the Firm". Economica 4 (16): 386-405.

- 1959. "The Federal Communications Commission". Journal of Law and Economics 2: 1-40.

— 1960. "The Problem of Social Cost". Journal of Law and Economics 3: 1-44.

—. 1974. "The Lighthouse in Economics". Journal of Law and Economics 17 (2): 357-76.

Demsetz, Harold. 1969. "Information and Efficiency: Another Viewpoint". Journal of Law and Economics 12 (1): 1-22.

Foldvary, Fred. 2003. "The Lighthouse as a Private-Sector Collective Good". En The Half-Life of Policy Rationales: How New Technology Affects Old Policy Issues, editado por Fred Foldvary \& Daniel Klein (New York: New York University Press). http:/www.independent.org/publications/working_papers/ article.asp?id=757/.

Lindberg, Erik. 2009. "The Market and the Lighthouse: Public Goods in Historical Perspective". Presentación en la Economic History Society Annual Conference, 3-5 de abril, University of Warwick.

Mill, John Stuart. (1848) 1909. Principles of Political Economy with some of their Applications to Social Philosophy, $7^{\mathrm{a}}$ ed. (London: Longmans Green \& Co.). http://www.econlib.org/library/Mill/mlPCover.html. 
Põder, Kare. 2010. "The Lighthouse in Estonia: The Provision Mechanism of 'Public Goods"'. En Discussions on Estonian Economic Policy XVIII, editado por Matti Raudjärv, 323-347. Berlin: Berliner Wissenschafts-Verlag. http:// www.mattimar.ee/publikatsioonid/majanduspoliitika/2010/2010.pdf

Samuelson, Paul A. 1954. "The Pure Theory of Public Expenditure". The Review of Economics and Statistics 36 (4): 387-389.

Trinity House. “A Brief History of Trinity House”. http://www.trinityhouse.co.uk/ pdfs/info-sheets/a_brief_history_of_trinity_house.pdf.

Van Zandt, David E. 1993. "The Lessons of the Lighthouse: 'Government' or 'Private' Provision of Goods”. The Journal of Legal Studies 22 (1): 47-72. EP 